2010-01-01

\title{
Polarization Dependence of an Edge Filter Based on Singlemode- Multimode-Singlemode Fibre
}

\author{
Agus Hatta \\ Technological University Dublin, ahatta@tudublin.ie \\ Yuliya Semenova \\ Technological University Dublin, yuliya.semenova@tudublin.ie \\ Ginu Rajan \\ Technological University Dublin, ginu.rajan@tudublin.ie
}

See next page for additional authors

Follow this and additional works at: https://arrow.tudublin.ie/engscheceart

\section{Recommended Citation}

Hatta, A. et al. (2010) Polarization Dependence of an Edge Filter Based on Singlemode-MultimodeSinglemode Fibre. Optics \& Laser Technology, Vol. 42, no. 6, September, pp. 1044-1048 doi:10.1016/ j.optlastec.2010.01.028

This Article is brought to you for free and open access by the School of Electrical and Electronic Engineering at ARROW@TU Dublin. It has been accepted for inclusion in Articles by an authorized administrator of ARROW@TU Dublin. For more information, please contact arrow.admin@tudublin.ie, aisling.coyne@tudublin.ie, gerard.connolly@tudublin.ie.

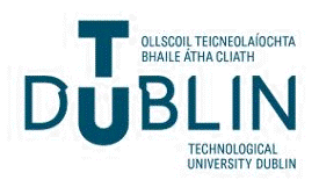




\section{Authors}

Agus Hatta, Yuliya Semenova, Ginu Rajan, and Gerald Farrell

This article is available at ARROW@TU Dublin: https://arrow.tudublin.ie/engscheceart/71 


\title{
Polarization dependence of an edge filter based on singlemode-multimode-singlemode fibre
}

\author{
A. M. Hatta ${ }^{1,2, *}$, Y. Semenova ${ }^{1}$, G. Rajan $^{1}$ and G. Farrell ${ }^{1}$ \\ ${ }^{1}$ Photonics Research Centre, School of Electronic and Communications Engineering, \\ Dublin Institute of Technology, Kevin Street, Dublin 8, Ireland \\ ${ }^{2}$ On leave from Engineering Physics Department, Institut Teknologi Sepuluh \\ Nopember (ITS), Surabaya, Indonesia \\ *Corresponding author. Address: Photonics Research Centre, School of Electronic \\ and Communications Engineering, Dublin Institute of Technology, Kevin Street, D8, \\ Ireland. Tel: +35314024575 \\ E-mail address: ahatta@dit.ie
}

\begin{abstract}
The polarization dependent loss (PDL) of a singlemode-multimodesinglemode (SMS) fibre structure used as an edge filter is presented. Minor errors in the fabrication process for the SMS fibre structure can introduce SMS fibre core offsets. The PDL due to lateral and rotational core offsets is investigated numerically and experimentally. It is shown that small core offsets are necessary to achieve low PDL for a SMS fibre based edge filter. It is also demonstrated that when lateral core offsets are unavoidable, the PDL of a SMS edge filter can still be minimized by introducing a rotational core offset of $90^{\circ}$.
\end{abstract}

Keywords: multimode fibre, polarization dependent loss, edge filter

\section{Introduction}

One approach to measuring optical wavelength is the use of a ratiometric all-fibre scheme, with the advantages of low cost, simple configuration, simple interconnections and the potential for high speed measurement. An all-fibre ratiometric wavelength measurement scheme consists of a $3 \mathrm{~dB}$ coupler with the two coupler outputs connected to a fibre edge filter arm, with a well defined spectral response, and a reference arm. Alternatively, two fibre edge filters arms with overlapping and opposite slope spectral responses, a so-called X-type spectral response, can be used. The use of an X-type spectral response can increase the measurement resolution of the ratiometric system [1]. Two edge filters for an X-type spectral response can be implemented by using step index singlemode-multimodesinglemode (SMS) fibre structures [2].

A SMS fibre structure also has been demonstrated for applications such as a bandpass filter, strain and temperature sensors, a wavelength encoded temperature sensor, and an intensity based temperature sensor [3-6]. The SMS fibre structure is fabricated by splicing a specified length of a multimode fibre (MMF) between two singlemode fibres (SMF). A commercial fibre fusion splicer is normally used to splice SMF to SMF or MMF to MMF with a very low loss, which means very low lateral core offsets. However fusion splicers are not pre-programmed to deal with splicing SMF to MMF so that during the splicing process for SMF to MMF or vice-versa, significant lateral core offset errors may arise. In a previous study [7], it is shown that an upper limit to lateral core offset is needed in order to ensure the edge filter spectral response stays within specification. 
It is well known, that individually SMF or MMF has a low polarization dependent loss (PDL), but for a SMS fibre structure, the PDL for an edge filter application has not been investigated. It has been previously shown that the polarization dependence of the edge filter based on a macro bend fibre in the all-fibre ratiometric system has a significant effect, specifically that high PDL for an edge filter can significantly decrease the accuracy of wavelength measurement [8]. In this paper the PDL of an SMS edge filter is investigated numerically and experimentally, in particular the PDL induced by lateral core offsets.

\section{Calculation of PDL for a SMS fibre structure}

The SMS fibre structure is shown in the inset figure in Fig. 1. It is formed by splicing a step-index multimode fibre (MMF) between two standard singlemode fibres (SMF). A brief review of the design, fabrication, and characterisation of a SMS edge filter can be found in [2]. For a MMF length of $44.44 \mathrm{~mm}$, with a core/cladding diameter of $9 / 125 \mu \mathrm{m}$ for the SMF and 105/125 $\mu \mathrm{m}$ for the MMF is shown in Fig. 1. A negative slope edge filter response can be obtained in a wavelength range of about $20 \mathrm{~nm}$ from 1505 to $1525 \mathrm{~nm}$.

Fig. 1 Schematic structure of a SMS fibre structure (inset). Calculated spectral response of SMS fibre structure.

To investigate polarization dependent effects, a modal propagation analysis (MPA) is performed in the Cartesian coordinate system with a set of calculated guided modes using the finite difference method (FDM) [7]. It should be noted that FDM calculates a set of all possible guided modes and can be calculated for quasi TE $\left(E_{x}\right)$ and quasi TM $\left(E_{y}\right)$ modes, allowing investigation of polarization effects. The PDL is defined as the difference in the transmission loss $(L s)$ between the quasi TE and quasi TM modes in $\mathrm{dB}$ as

$$
P D L=\left|L s_{T E}-L s_{T M}\right| .
$$

The splicing process can introduce lateral core offsets between the SMS fibre cores, that is the input/output SMFs may have lateral core offsets relative to the centre of the MMF core. In addition the two lateral core offsets at each end of the MMF may also have a different orientation relative to each other, which will henceforth be referred to as a rotational offset. In order to analyze the PDL of a SMS fibre structure, a means to precisely describe lateral and rotational offsets is needed. Fig. 2(a) and Fig. 2(b) show the interfaces between the input SMF and the MMF section cores and the MMF section and the output SMF cores, respectively. Where the lateral core offsets of the input and output SMF have the same orientation, the rotational core offset is defined as $0^{\circ}$. Rotational core offsets of $90^{\circ}$ and $180^{\circ}$ are also shown in Fig. 2(b).

Fig. 2 Interfaces of input/output SMF core to the MMF core (a) position of input SMF core, and (b) position of output SMF core.

The PDL of a SMS edge filter due to the lateral and rotational core offset is calculated using (1). Fig. 3 shows the PDL at a wavelength of $1510 \mathrm{~nm}$ for a rotational core offset from 0 to $180^{\circ}$ for lateral core offset values of $1,2,3$, and $4 \mu \mathrm{m}$. The limit of $4 \mu \mathrm{m}$ is chosen because in [7] it is shown that the spectral responses of a SMS edge filter degrades significantly when the lateral core offsets exceeds the SMF 
core radius. One can see that the PDL depends on the lateral and rotational core offsets. Generally, a larger lateral core offset induces a higher PDL. However, it is clear that the PDL at the rotational core offset of $90^{\circ}$ has the lowest PDL for the lateral core offsets from 1 to $4 \mu \mathrm{m}$.

Fig. 3 Calculated PDL for several lateral core offsets at the rotational core offset from 0 to $180^{\circ}$.

The physical insight into the results in Fig. 3 is as follows. Assuming some lateral core offset of the input SMF is as in Fig. 2(a), the field profile at the output end of the MMF section depends on the input field polarization state of the quasi TE mode (x-directed) or quasi TM mode (y-directed). In turn, the transmission loss for each mode depends on the overlap between the field profile at the output end of the MMF section and the eigen-mode profile of the output SMF. A low PDL occurs when the overlap profiles for the TE and TM modes are similar. At a rotational core offset of $90^{\circ}$, the orientation between the input/output SMF and the input field direction of TE/TM are parallelized. Thus the overlap between the field profile at the output end of the MMF section and the eigen-mode profile of the output SMF for both TE and TM modes are similar and the PDL is minimized.

To illustrate this insight further, the field amplitude profiles at the output end of the MMF section when the input SMF has a lateral core offset of $4 \mu \mathrm{m}$ are shown in Fig. 4(a) and Fig. (b) for TE and TM modes, respectively. Fig. 4(c) and Fig. 4(d) are the same profiles but with greater magnification, in the vicinity of the output SMF position. It can be seen from the magnified images that the amplitude profiles for the TE and TM modes are slightly different, and this difference in the amplitude profiles is presented in Fig. 4(e). It is clear that the difference in the amplitude for TE and TM modes varies significantly with the co-ordinates within the cross-section of the output end of the MMF. One can see that in the regions corresponding to $90^{\circ}$ or $270^{\circ}$ rotational offsets the difference between TE and TM amplitudes is lower compared to the positions corresponding to 0 and $180^{\circ}$ rotational offsets as in Fig. 3. Thus when the output SMF is positioned so that its core centre is at the point of minimal difference and thus has a rotational core offset of $90^{\circ}$, the PDL is minimized.

Therefore during the fabrication of a SMS edge filter it is desirable to have a low lateral core offsets but should lateral offsets occur, rotating the output SMF relative to the input SMF by $90^{\circ}$ can mitigate the effect on PDL of lateral offset. The resultant PDL of the SMS structure is minimized.

Fig. 4 Field amplitude profile at the output end of the MMF section (a) TE mode, (b) TM mode; close up images: (c) TE mode, (d) TM mode, and (e) the difference in the amplitude profiles between TE and TM modes.

\section{Experimental results}

The SMS edge filter described above was fabricated using a precision Fujikura CT-07 cleaver and a Sumitomo type-36 three-axis fusion splicer. Four edge filters based on SMS structures were fabricated to investigate the PDL due to the lateral and rotational core offsets.

Firstly, two edge filters, SMS-1 and SMS-2 were fabricated using an automatic splicing mode where there is no user control of lateral and rotational cores offsets. The transmission responses of the fabricated SMS edge filters were measured 
using a tunable laser TUNIC PLUS and a power meter as shown in Fig. 5(a). It is clear that the wavelength range of 1505 to $1525 \mathrm{~nm}$ is suitable for an edge filter application. The MMF length of SMS-1 and SMS-2 is circa $44.4 \mathrm{~mm} \pm 0.2 \mathrm{~mm}$, corresponding to $\mp 8 \mathrm{~nm}$ shifts in the spectral response [9]. The lateral core offsets are circa 0.5 to $1 \mu \mathrm{m}$ according to the fusion splicer's post splicing report.

To measure the PDL of the SMS edge filters, a fibre polarization controller was used to change the polarization state of the input signal. The SMS fibre structures were fixed to a rigid base using super glue, to prevent bending, twisting and strain effects of the SMS structure. The transmission response was measured and the difference between the maximum and minimum transmission response was calculated as the PDL. The PDL of the fabricated SMS edge filters were measured within the wavelength range with an increment of $2.5 \mathrm{~nm}$ as shown in Fig. 5(b). The PDL of SMS-1 and SMS-2 produced using the automatic splicing mode shows an average PDL of 0.088 and $0.112 \mathrm{~dB}$, respectively. A standard deviation of the average PDL within the wavelength range is $\pm 1.685 \times 10^{-3}$ and $\pm 1.886 \times 10^{-3}$ for SMS-1 and SMS-2, respectively. The measured PDL results include the inherent PDL of the system which is about $0.03 \mathrm{~dB}$.

Fig. 5 Measured results of SMS edge filters using the automatic splicing mode (a) spectral responses, (b) PDL.

Secondly, SMS-3 and SMS-4, with MMF lengths of about $44.4 \mathrm{~mm}$ were fabricated using an attenuation splicing mode with the same lateral core offsets as in the case of the auto mode, that is $3.31 \mu \mathrm{m}$, but with different rotational core offsets of $90^{\circ}$ and $180^{\circ}$, respectively. A given lateral offset can be achieved using the splice attenuation setting of the fusion splicer. A splicing attenuation of $1 \mathrm{~dB}$, which is the minimum value, corresponds to a lateral core offset of $3.31 \mu \mathrm{m}$. Fig. 6 shows a screenshot in the vicinity of the output splice, using the attenuation splicing setting. The screenshot shows the MMF and SMF (M-S structure) on the left and right side, respectively.

To achieve different rotational offsets in our experiments the input splice for the SMS has a lateral core offset applied prior to fusion such that the input SMF is shifted in the y-axis by $3.31 \mu \mathrm{m}$ as in Fig. 2(a). Fusion is then carried out but before the spliced fibres are removed from the splicing machine the top of the input SMF is carefully marked. To splice the other end of the MMF, the S-M structure is again placed in the splicing machine, with a y-axis shift of $3.31 \mu \mathrm{m}$. However the rotational position is controlled by rotating the $\mathrm{S}-\mathrm{M}$ structure from the reference marker to the desired rotational core offset.

Fig. 6 Screenshot of the splicing process using attenuation splicing mode.

The transmission responses of SMS-3 and SMS-4 were measured as shown in Fig. 7(a). The wavelength range of 1505 to 1525 is suitable for an edge filter application. The measured PDL of SMS-3 and SMS-4 is shown in Fig. 7(b). The average PDL of SMS-3 and SMS-4 for the rotational core offset of $180^{\circ}$ and $90^{\circ}$ are 0.215 and $0.092 \mathrm{~dB}$, respectively. The standard deviation of the average PDL within the wavelength range is $\pm 1.733 \times 10^{-3}$ and $\pm 1.423 \times 10^{-3}$ for SMS-3 and SMS-4, respectively. It is clear from Fig. 7(b) that the average PDL of SMS edge filter decreases noticeably for the case of a rotational core offset of $90^{\circ}$. It is also clear from 
Fig. 5(b) and Fig. 7(b), that the average PDL depends on the lateral offsets, where a lower lateral core offset exhibits a lower PDL.

It can be concluded that the main source of PDL for a SMS edge filter is lateral core offset. Where lateral core offsets do exist, the value of the rotational core offset can either increase or decrease the net PDL. Therefore, in order to minimize the PDL, it is necessary to ensure low lateral core offsets but if lateral offsets cannot be avoided then control of the rotational core offset is needed during the fabrication of a SMS edge filter. It is also preferable to use a three axis adjustment fibre fusion splicer instead of a single axis adjustment (a fixed V-grove) fibre fusion splicer. Using a three axis adjustment splicer can minimize the lateral offsets that arise because of the limited core-cladding concentricity of the fibres and in turn can minimize both the overall loss and the PDL.

Fig. 7 Measured results of SMS edge filters with the rotational core offsets of $180^{\circ}$ and $90^{\circ}$ (a) spectral responses, (b) PDL.

\section{Conclusion}

The PDL of a SMS fibre based edge filter has been investigated. A MPA based on FDM is used to analyze the PDL of SMS edge filter with core offsets. It is demonstrated that the PDL of the SMS edge filter depends on its lateral and rotational core offsets. Lateral core offsets are undesirable as they will increase the PDL for the SMS edge filter. However, if lateral offsets do occur, then by introducing a rotational core offset of $90^{\circ}$, the PDL can be minimized considerably.

\section{References}

[1] S. M. Melle, K. Liu, and R. M. Measures, Practical fibre-optic Bragg grating strain gauge system, App. Opt. 32 (1993) 3601-3609.

[2] A. M. Hatta, G. Farrell, Q. Wang, G. Rajan, P. Wang, and Y. Semenova, Ratiometric wavelength monitor based on singlemode-multimode-singlemode fibre structure, Microwave and Opt. Technol. Lett. 50 (2008) 3036-3039.

[3] W. S. Mohammed, P. W. E. Smith, and X. Gu, All-fibre multimode interference bandpass filter, Opt. Lett. 31 (2006) 2547-2549.

[4] D. P. Zhou, L. Wei, W. K. Liu, Y. Liu, and J. W. Y. Lit, Simultaneous measurement for strain and temperature using fibre Bragg gratings and multimode fibres, App. Opt. 47 (2008) 1668-1672.

[5] E. Li, G.-D. Peng, Wavelength-encoded fibre-optic temperature sensor with ultrahigh sensitivity, Opt. Commun. 281 (2008) 5768-5770.

[6] A. M. Hatta, G. Rajan, Y. Semenova and G. Farrell, A SMS fibre structure for temperature measurement using a simple intensity based interrogation system, Electron. Lett. 45 (2009) 1069-1071.

[7] A. M. Hatta, G. Farrell, P. Wang, G. Rajan, and Y. Semenova, Misalignment limits for a singlemode-multimode-singlemode fibre based edge filter, J. Lightwave Technol. 27 (2009) 2482-2488.

[8] G. Rajan, Q. Wang, Y. Semenova, G. Farrell P. Wang, Effect of polarization dependent loss on the performance accuracy of a ratiometric wavelength measurement system, IET Optoelectronics 2 (2008) 63-68.

[9] Q. Wang, G. Farrell, and W. Yan, Investigation on singlemode-multimodesinglemode fibre structure, J. Lightwave Technol. 26 (2008) 512-519. 


\section{Figure Captions}

Fig. 1 Schematic structure of a SMS fibre structure (inset). Calculated spectral response of SMS fibre structure.

Fig. 2 Interfaces of input/output SMF core to the MMF core (a) position of input SMF core, and (b) position of output SMF core.

Fig. 3 Calculated PDL for several lateral core offsets at the rotational core offset from 0 to $180^{\circ}$.

Fig. 4 Field amplitude profile at the output end of the MMF section (a) TE mode, (b) TM mode; close up images: (c) TE mode, (d) TM mode, and (e) the difference in the amplitude profiles between TE and TM modes.

Fig. 5 Measured results of SMS edge filters using the automatic splicing mode (a) spectral responses, (b) PDL.

Fig. 6 Screenshot of the splicing process using attenuation splicing mode.

Fig. 7 Measured results of SMS edge filters with the rotational core offsets of $180^{\circ}$ and $90^{\circ}$ (a) spectral responses, (b) PDL. 


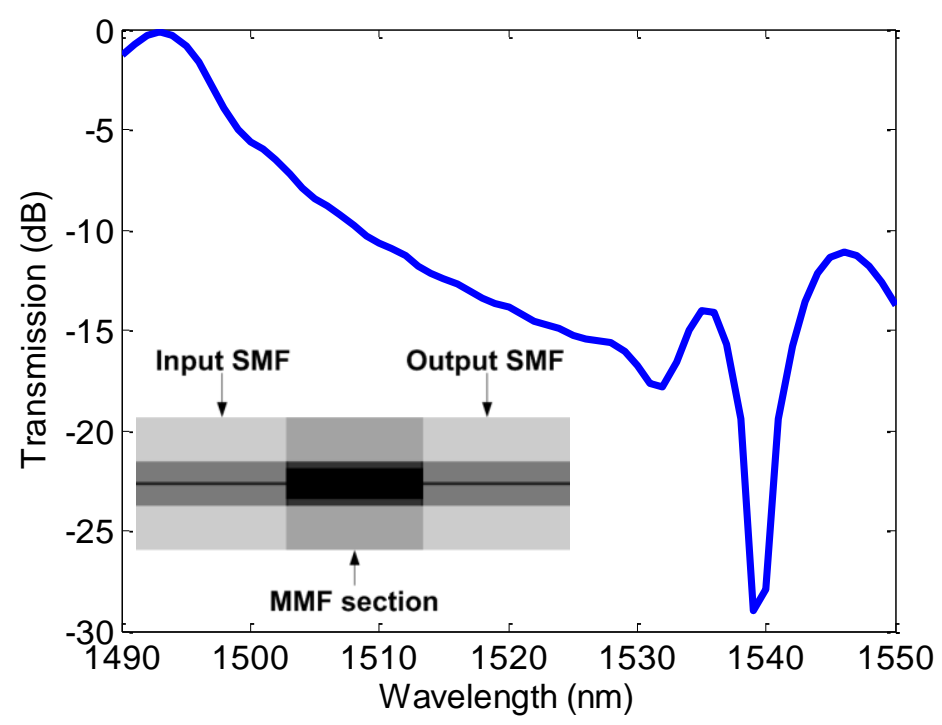

Fig. 1 Schematic structure of a SMS fibre structure (inset). Calculated spectral response of SMS fibre structure. 


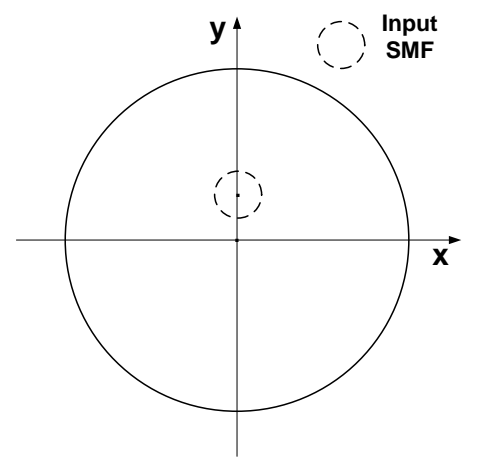

(a)

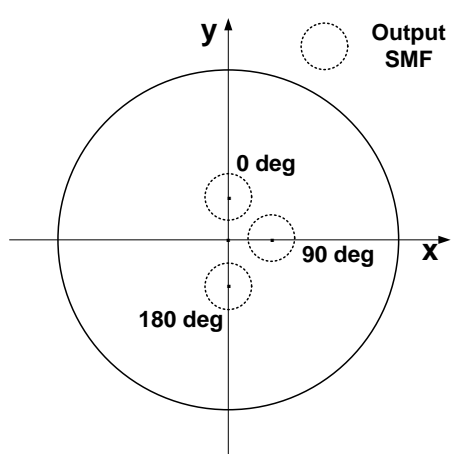

(b)

Fig. 2 Interfaces of input/output SMF core to the MMF core (a) position of input SMF core, and (b) position of output SMF core. 


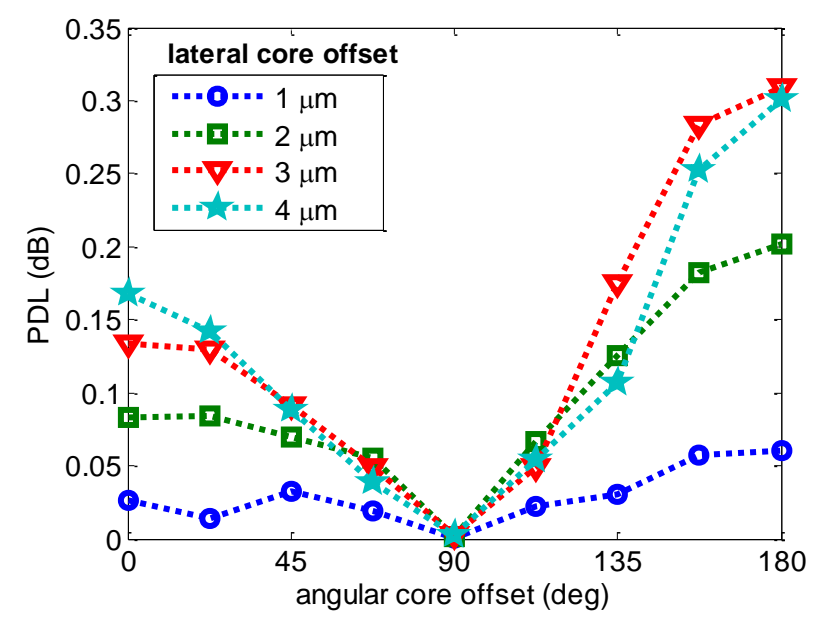

Fig. 3 Calculated PDL for several lateral core offsets at the rotational core offset from 0 to $180^{\circ}$. 


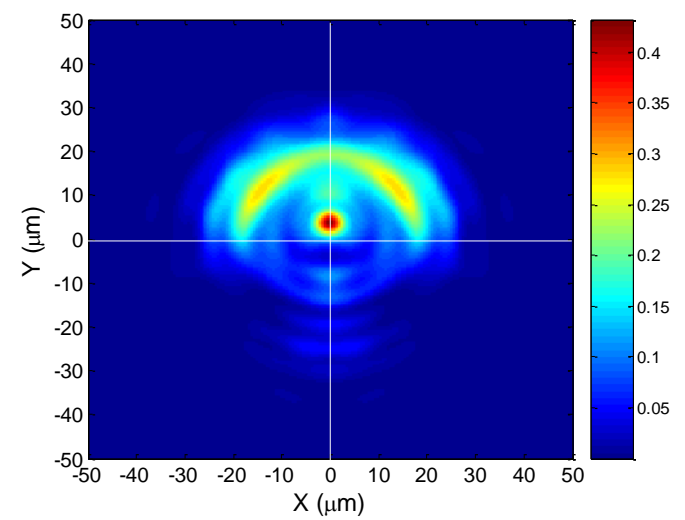

(a)

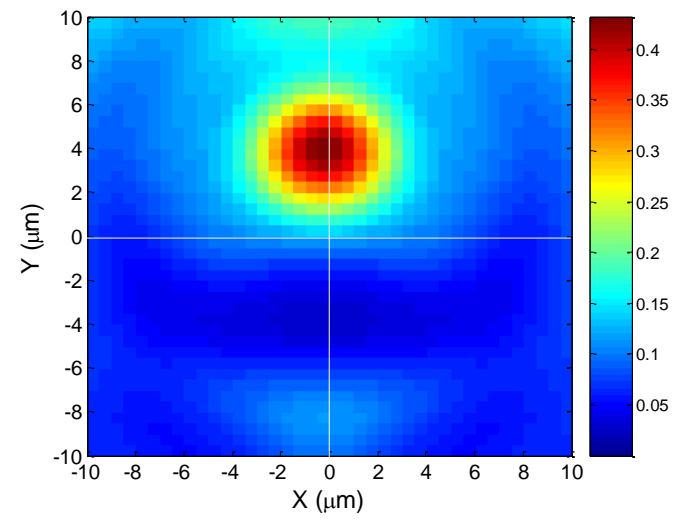

(c)

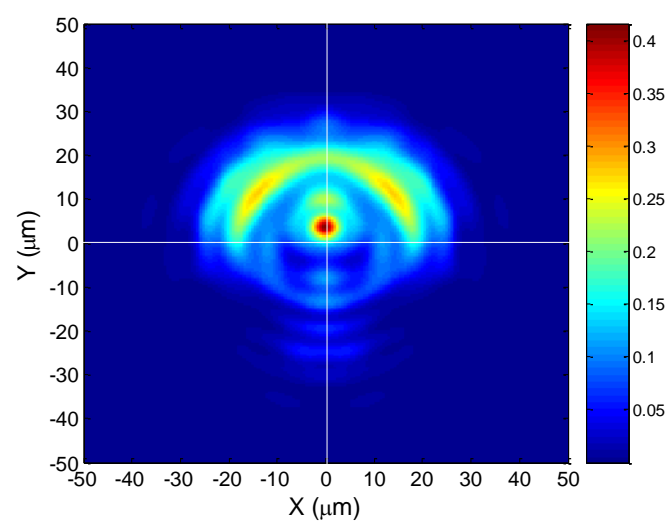

(b)

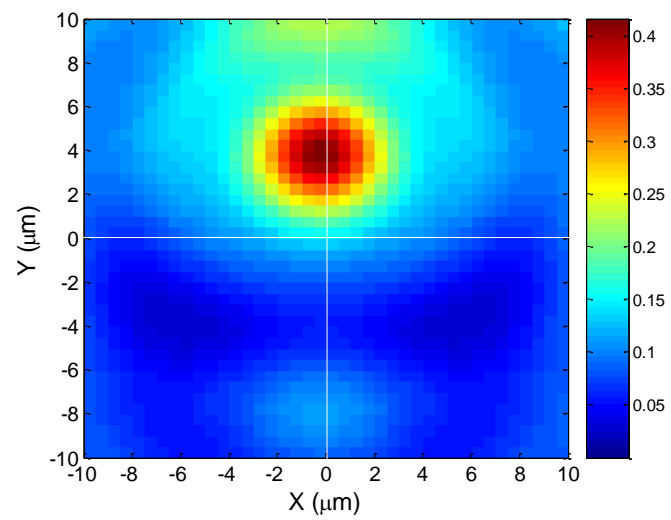

(d)



(e)

Fig. 4 Field amplitude profile at the output end of the MMF section (a) TE mode, (b) TM mode; close up images: (c) TE mode, (d) TM mode, and (e) the difference in the amplitude profiles between TE and TM modes. 


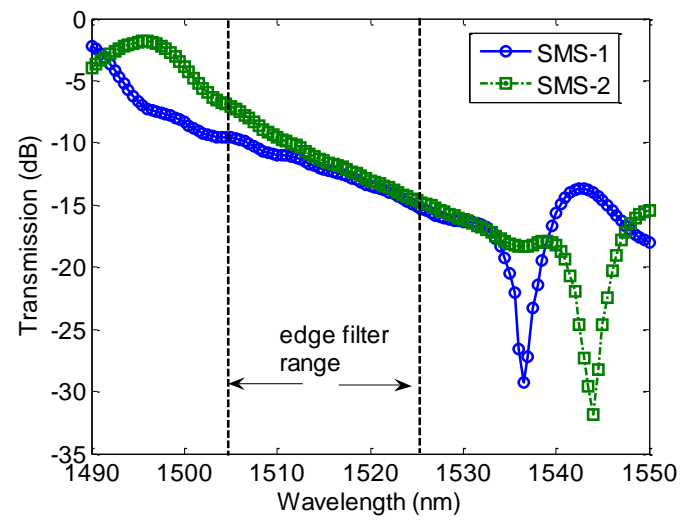

(a)

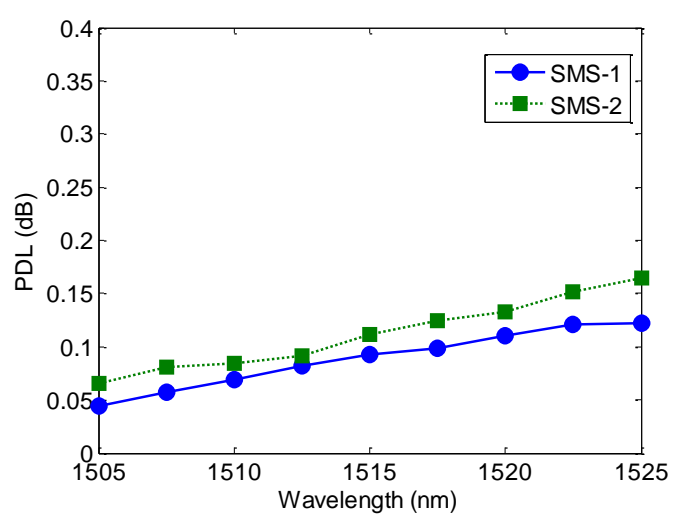

(b)

Fig. 5 Measured results of SMS edge filters using the automatic splicing mode (a) spectral responses, (b) PDL. 


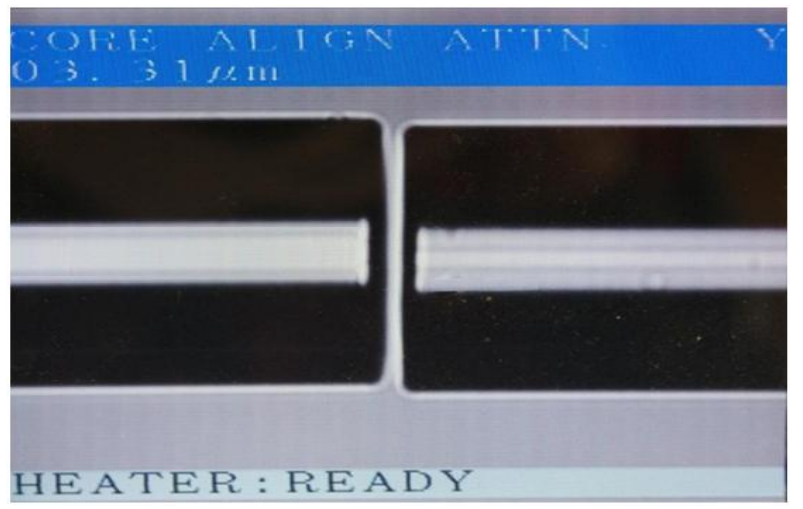

Fig. 6 Screenshot of the splicing process using attenuation splicing mode. 


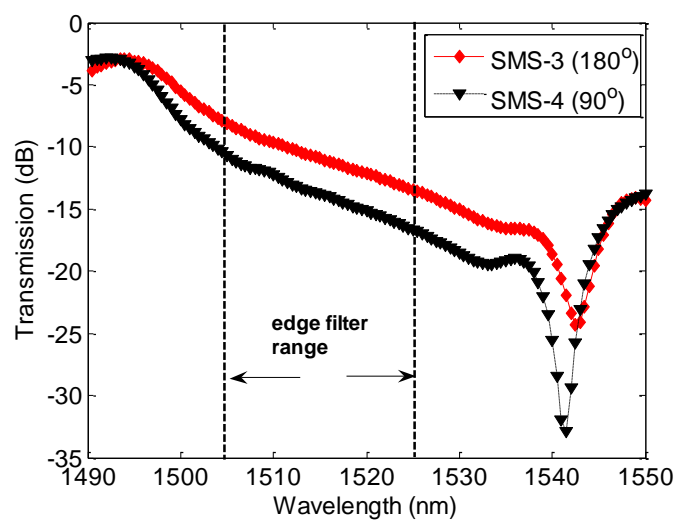

(a)

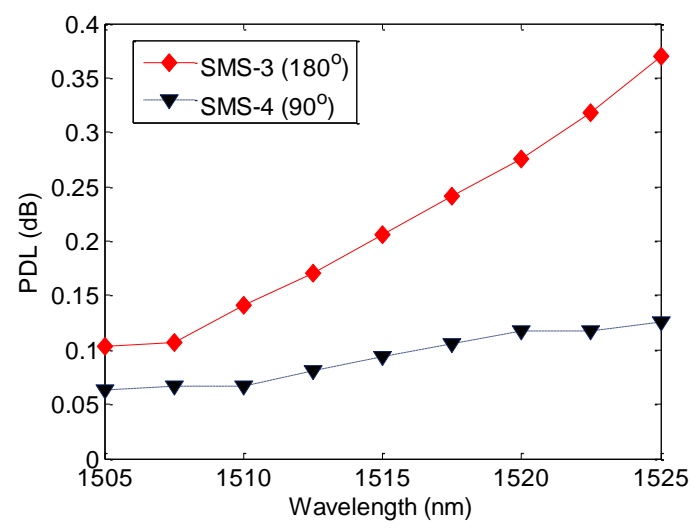

(b)

Fig. 7 Measured results of SMS edge filters with the rotational core offsets of $180^{\circ}$ and $90^{\circ}$ (a) spectral responses, (b) PDL. 